\title{
Anomalous scaling laws of intensity fluctuations in the solar photosphere ${ }^{\star}$
}

\author{
F. Lepreti ${ }^{\star \star}$ \\ Dipartimento di Fisica, Università della Calabria, 87036 Rende (CS), Italy \\ Istituto Nazionale per la Fisica della Materia, Unità di Cosenza, Italy \\ Received 3 April 2001 / Accepted 3 August 2001

\begin{abstract}
The photospheric intensity field, obtained from THEMIS-IPM observations, is analysed. We investigate the scaling behavior of intensity fluctuations, following an approach which is used in the study of passive scalar fluctuations in fully turbulent flows. By means of the methods which are applied in the study of multifractal measures, it is shown that the intensity fluctuations are characterised by anomalous scaling laws, identified by defining a dissipation field in analogy with the dissipation of passive scalar fluctuations in fluids. It is shown, finally, that this field is more intermittent in the higher photospheric layers than in the lower ones.
\end{abstract}

Key words. Sun: photosphere

\section{Introduction}

The study of intensity and velocity fields in the solar photosphere is very important for the characterisation of many physical processes which occur in the solar atmosphere such as generation of $p$-mode oscillations and coronal heating (Stix 1991). In some previous works, based on the analysis of velocity and intensity Fourier spectra, it has been suggested that a turbulent cascade process could have a relevant role in the formation of the pattern, namely the granulation, which is visible in high resolution images of the photosphere (Espagnet et al. 1993; Salucci et al. 1994). However, this result is not in agreement with other studies based on observations (Deubner 1988) or numerical simulations (Nordlund et al. 1997; Stein \& Nordlund 1998).

The presence of scaling relations and intermittency phenomena in the photospheric intensity and vertical velocity fields have recently been shown (Consolini et al. 1999; Lepreti et al. 2000a,b). In there works, analytical and numerical tools, which had previously been used in modeling the fluid turbulence and the behavior of chaotic systems, have been applied to study the scaling properties of the photospheric structures. Following this approach, in the present paper we investigate the scaling laws of photospheric intensity fluctuations by means of the multifractal

* Based on observations made with THEMIS-CNRS/INSUCNR operated on the island of Tenerife by THEMIS S.L. in the Spanish Observatorio del Teide of the Instituto de Astrofisica de Canarias.

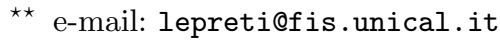

measure concept (Paladin \& Vulpiani 1987). In order to show the difference between the scaling behavior at different photospheric heights, we analyze monochromatic and white light images acquired simultaneously.

\section{Observations and data reduction}

The monochromatic and white light (WL) images analysed in this work were acquired on July 1, 1999 with the Italian Panoramic Monochromator (Cavallini 1998) installed at the THEMIS telescope. The observed field of view is about $30^{\prime \prime} \times 30^{\prime \prime}$ (corresponding to $222 \times 222$ pixels with a pixel size of $\left.0.134^{\prime \prime}\right)$, the exposure time is $200 \mathrm{~ms}$ and the spatial resolution is $\simeq 0.4^{\prime \prime}$. The observed quiet solar region is located at the disk center.

The monochromatic images were acquired at 7 wavelengths within the Fe I $557.61 \mathrm{~nm}$ absorption line. From this images, center line intensity fields were calculated after reconstructing the line profiles pixel by pixel from a best fit of the observed intensities. The spatial resolution of the center line images is about $0.8^{\prime \prime}$. The Fe I $557.61 \mathrm{~nm}$ absorption line is formed at a photospheric height of about $370 \mathrm{~km}$ (Komm et al. 1991) and it provide us with information about the structure of the higher layers of the photosphere.

Besides the standard corrections for instrumental and atmospheric effects, both the WL and the center line intensity fields were filtered by means of a subsonic filter to remove the contribution of the 5-min acoustic waves ( $p$-modes). The time series used for this procedure consist of 32 images which cover a time interval of about $40 \mathrm{~min}$. 

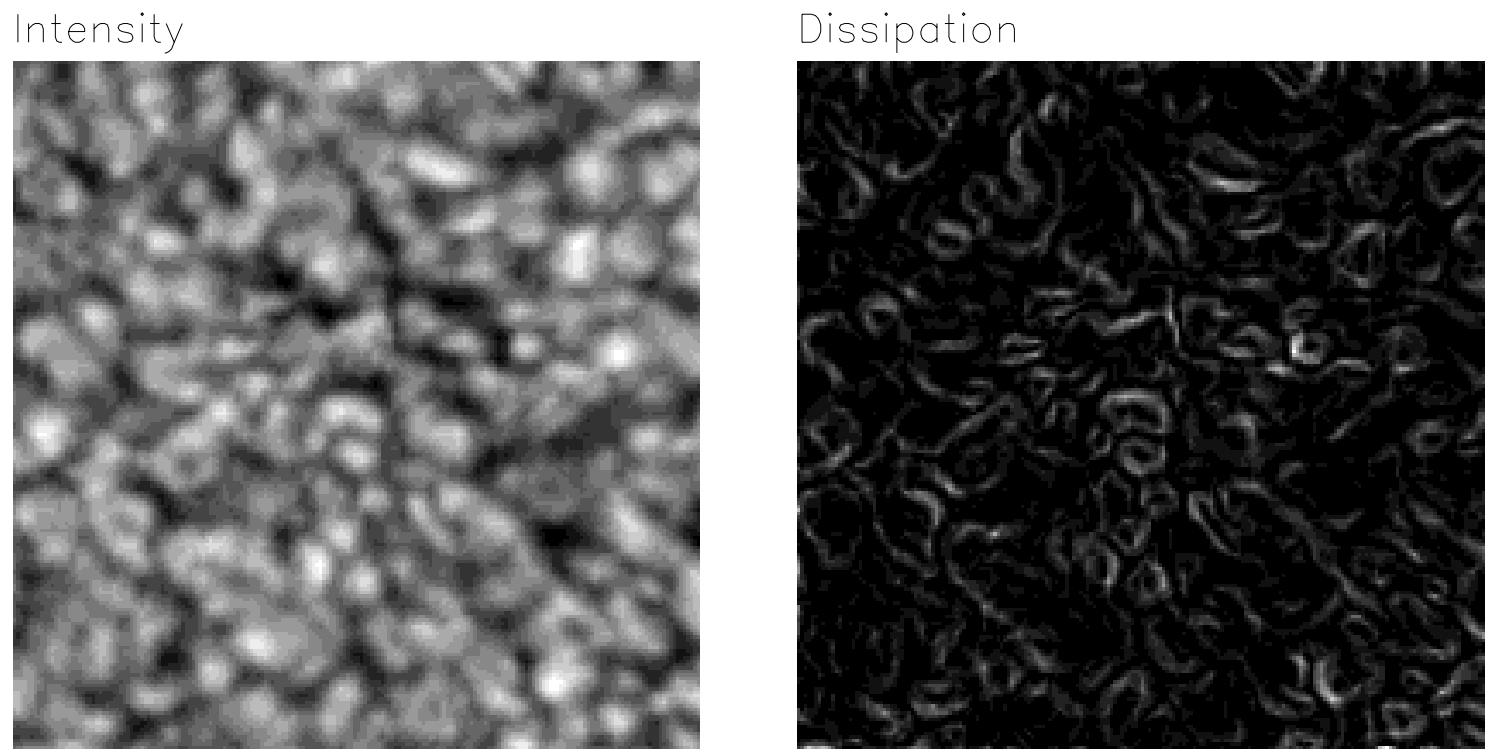

Fig. 1. An example of WL intensity field $\Gamma(x, y)$ (left panel) and the corresponding dissipation field (right panel).

\section{Data analysis}

The analysis of passive scalar fluctuations in fluids is an important tool to study the properties of transport in turbulent flows. In the past years it has been shown that the dissipation of passive scalars in fully developed turbulence is characterised by an intermittent behavior, which can be described by multifractal models (Prasad et al. 1988; Sreenivasan 1991).

In this paper, we try to make use of these concepts to study photospheric intensity fluctuations observed with the THEMIS-IPM system. If we denote by $I(x, y)$ the intensity field, we can define the "dissipation" field $\Gamma(x, y)$ as

$\Gamma(x, y) \simeq|\nabla I|^{2}=\left(\frac{\partial I}{\partial x}\right)^{2}+\left(\frac{\partial I}{\partial y}\right)^{2}$,

in analogy with the definition of the dissipation of passive scalars in fluids (Prasad et al. 1988). In Fig. 1 we show an example of WL image with the corresponding dissipation field $\Gamma(x, y)$. Comparing the two images, it can be noted that large variations in $\Gamma(x, y)$ occur at the boundaries of the granular cells. Moreover $\Gamma(x, y)$ is characterised by an intermittent structure, the fluctuations being concentrated at small spatial scales.

To compare the intermittency properties of the dissipation field at different photospheric heights, we can look for the presence of anomalous scaling laws following multifractal models (Paladin \& Vulpiani 1987; Sreenivasan 1991). To this end, we introduce a probability measure

$\varepsilon_{i}(r)=\frac{\chi_{i}(r)}{\chi(L)}$

where

$\chi_{i}(r)=\int_{D_{i}(r)} \Gamma(x, y) \mathrm{d} x \mathrm{~d} y$

is a coarse graining of $\Gamma(x, y)$ at the scale $r . D_{i}(r)$ represents a hierarchy of disjoint squares of size $r$ covering the

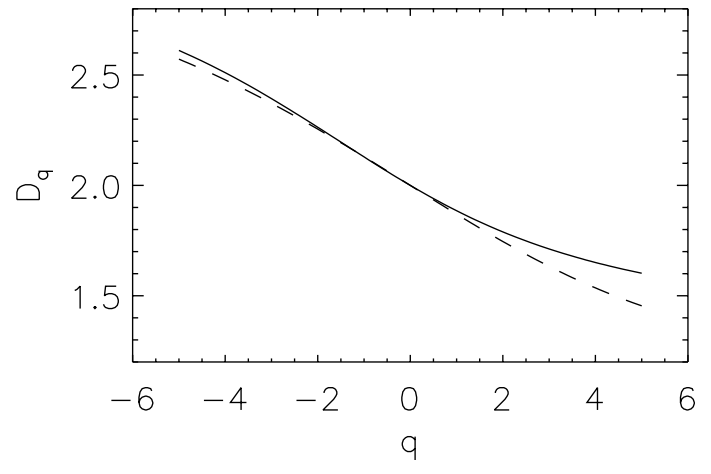

Fig. 2. The generalized dimensions $D_{q}$ for the scalar dissipation field obtained from WL intensity fields (solid line) and from Fe I $557.61 \mathrm{~nm}$ center line intensity fields (dashed line).

whole field of view $D(L)$. The scaling properties of $\Gamma(x, y)$ can be investigated by calculating the moments

$\left\langle\varepsilon^{q}\right\rangle=\sum_{i} \varepsilon_{i}^{q}(r)$

of the probability measure, and identifying power laws of the type

$\left\langle\varepsilon^{q}\right\rangle \sim r^{(q-1) D_{q}}$,

where $D_{q}$ represent the generalized dimensions (Hentschel \& Procaccia 1983).

We calculated the moments $\left\langle\varepsilon^{q}\right\rangle$ for $0.095 \mathrm{Mm} \leq r \leq$ $19.5 \mathrm{Mm}$ and $-5 \leq q \leq 5$. It's worthwhile to point out that the larger $q$ is the stronger the contribution given to $\left\langle\varepsilon^{q}\right\rangle$ by the most intermittent regions of the measure is, while for small values of $q$ the major contribution is due to the most regular regions.

If the probability measure is globally self-similar, $D_{q}$ is constant and it corresponds to the fractal dimension of the measure. On the contrary, if $D_{q}$ is not constant, the scaling laws are said to be anomalous and the measure 
can be described as a multifractal object. In this case, it can be shown also that $D_{q}$ is a nonincreasing function of $q . D_{0}$ corresponds to the dimension of the support of the measure, $D_{1}$ represents the information dimension, that is, the dimension of the set that contains the dominant contributions to the measure (Paladin \& Vulpiani 1987), while $D_{2}$ is the so-called correlation dimension (Grassberger \& Procaccia 1983).

In the plots of $\log \left\langle\varepsilon^{q}\right\rangle$ vs. $\log (r)$, we found a region of quite good scaling between $r=0.8 \mathrm{Mm}$ and $r=10 \mathrm{Mm}$. From the slope of these curves, we obtained the dimensions $D_{q}$ both for the WL and for the Fe I line core intensity fields. From the curves $D_{q}$ vs. $q$, which are shown in Fig. 2, we can see that, in both cases, the probability measure is characterised by a multifractal structure. In our case $D_{0}=2$ trivially corresponds to the dimension of the field domain, $D_{1} \simeq 1.88$ both for WL and Fe I images, while a slight difference is found for the correlation dimension, being $D_{2}=1.79 \pm 0.02$ for WL images and $D_{2}=1.75 \pm 0.02$ for Fe I images. Indeed, the most interesting information we can obtain comparing the two curves is that $\Gamma(x, y)$ is characterised by a more intermittent behavior in the higher photospheric layers than in the lower ones, as it can be argued from the fact that for positive values of $q, D_{q}$ is smaller for the Fe I line intensity field.

An equivalent description of the multifractal structure of $\varepsilon(r)$ can be given through the singularity spectrum $f(\alpha)$, that is the fractal dimension of the set of points in which the probability measure follows the scaling law

$\varepsilon(r) \sim r^{\alpha}$

It can be shown that the generalized dimensions are related to the singularity spectrum by the Legendre transform (Halsey et al. 1986)

$\alpha(q)=\frac{\mathrm{d}}{\mathrm{d} q}\left[(q-1) D_{q}\right]$

$f[\alpha(q)]=q \alpha-(q-1) D_{q}$.

The exponent $\alpha$ depends on the position and it can be related to the different strengths of local singularities present in the probability measure. In other words, the values $\alpha<d$ (where $d$ is the topological dimension, in our case $d=2$ ) occur in regions where the measure density diverges as $r \rightarrow 0$, with smaller $\alpha$ values corresponding to stronger singularities. On the contrary, the values $\alpha>d$ occur in regular regions of the measure. The whole measure can be considered as the superposition of different fractal sets, each characterised by a scaling exponent $\alpha$ and by the fractal dimension $f(\alpha)$.

The $f(\alpha)$ curves obtained from the WL images and from the Fe I center line images are shown in Fig. 3. It can be seen that the two curves differ for $\alpha<2$, reflecting the difference between the $D_{q}$ curves for $q>0$. This result is at variance with the case of passive scalar fluctuations in fully turbulent flows, which show a universal character

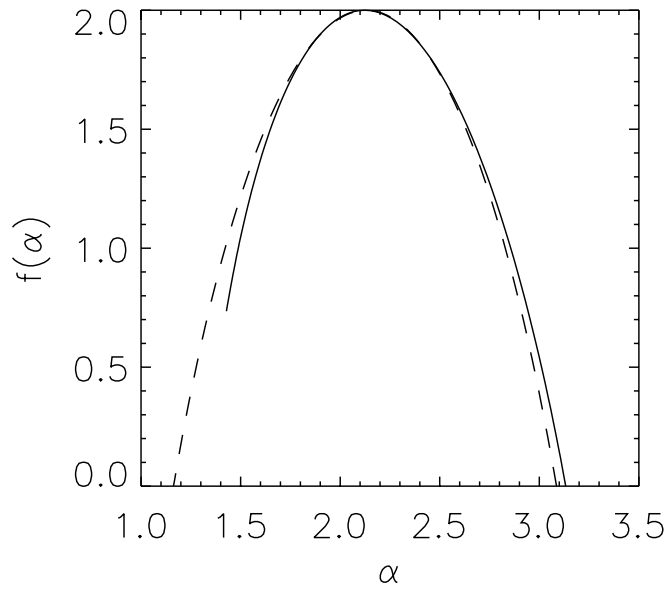

Fig. 3. The singularity spectrum $f(\alpha)$ for the scalar dissipation field calculated from WL intensity fields (solid line) and from Fe I $557.51 \mathrm{~nm}$ center line intensity fields.

in the sense that the singularity spectrum is the same for different scalar quantities provided that they are passive and that the Reynolds number is sufficiently high (Prasad et al. 1988).

It is worthwhile to point out that, at variance with earlier analyses (Lepreti et al. 2000b), the possibility to analyze simultaneous monochromatic and WL images and to filter the $p$-mode contribution in a more efficient way, allowed us to show in a more reliable way the difference in the scaling behavior between low and high photospheric layers.

\section{Conclusion}

In this paper we have studied the structure of intensity fluctuations at two different heights in the solar photosphere, trying to approach the problem from the point of view of dynamical system theory. To this aim, starting from photospheric intensity fields, we have defined a dissipation field in analogy with the dissipation of passive scalar fluctuations in turbulent flows and then we have investigated the occurrence of anomalous scaling laws in a suitable probability measure obtained from the dissipation field.

Even if the motions in the solar photosphere don't exhibit a fully turbulent behavior because of the presence of strong density stratification, this method can be useful to better understand the variation of the photospheric intensity pattern with the height. Due to the peculiar structure of the photospheric intensity pattern, we could expect, indeed, the intermittent character of the dissipation field, the strongest fluctuations being present at the boundaries of the cells.

On the other hand, the most interesting result which has been obtained in this work is the different behavior in the scaling laws for the WL images and for the Fe I center line images respectively. This indicates that the scalar dissipation field is characterised by stronger singularities in the higher photospheric layers than in the lower ones. In other words, the relative weight of large 
intensity fluctuations seems to increase with the height in the solar photosphere. This result can be related to the occurrence of some intensity features observed in previous works at high photospheric levels (Salucci et al. 1994; Espagnet et al. 1995). Other analysis methods are now under way to investigate the spatiotemporal behavior of photospheric structures, in order to better understand the origin of this phenomenon (Carbone et al. 2001).

Acknowledgements. The author is grateful to F. Berrilli, V. Carbone, G. Consolini and E. Pietropaolo for their help in data analysis and for useful suggestions. Thanks are due also to the THEMIS staff for efficient support in the observations.

\section{References}

Carbone, V., Lepreti, F., Primavera, L., et al. 2001, A\&A, in press

Cavallini, F. 1998, A\&A, 128, 589

Consolini, G., Carbone, V., Berrilli, F., et al. 1999, A\&A, 344, L33

Deubner, F.-L. 1988, A\&A, 204, 301

Espagnet, O., Muller, R., Roudier, T., \& Mein, N. 1993, A\&A, 271,589
Espagnet, O., Muller, R., Roudier, T., Mein, N., \& Mein, P. 1995, A\&AS, 109, 79

Grassberger, P., \& Procaccia, I. 1983, Phys. Rev. Lett., 50, 346

Halsey, T. C., Jensen, M. H., Kadanoff, L. P., Procaccia, I., \& Shraiman, B. I. 1986, Phys. Rev. A, 33, 1141

Hentschel, H. G. E., \& Procaccia, I. 1983, Physica D, 8, 435

Komm, R., Mattig, W., \& Nesis, A. 1991, A\&A, 252, 812

Lepreti, F., Carbone, V., Pietropaolo, E., et al. 2000a, Physica A, 280, 87

Lepreti, F., Bavassano, B., Berrilli, F., et al. 2000b, in Advances in Turbulence, Proceedings of the 8th European Turbulence Conference, ed. C. Dopazo (CIMNE Publications, Barcelona), 843

Nordlund, Å, Spruit, H. C., Ludwig, H.-G., \& Trampedach, R. 1997, A\&A, 328, 229

Paladin, G., \& Vulpiani, A. 1987, Phys. Rep., 156, 147

Prasad, R. R., Meneveau, C., \& Sreenivasan, K. R. 1988, Phys. Rev. Lett., 61, 74

Salucci, G., Bertello, L., Cavallini, F., Ceppatelli, G. \& Righini, A. 1994, A\&A, 285, 322

Sreenivasan, K. R. 1991, Ann. Rev. Fluid. Mech., 23, 539

Stein, R. F., \& Nordlund, A. 1998, ApJ, 499, 914

Stix, M. 1991, The Sun: An introduction (Springer Verlag, Berlin) 